Matthias Dahms

Motivieren, Delegieren, Kritisieren 
Matthias Dahms

\section{Motivieren, Delegieren, Kritisieren}

Die Erfolgsfaktoren der Führungskraft

2. Auflage

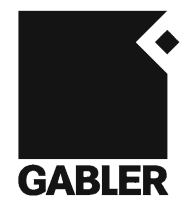


Bibliografische Information der Deutschen Nationalbibliothek

Die Deutsche Nationalbibliothek verzeichnet diese Publikation in der

Deutschen Nationalbibliografie; detaillierte bibliografische Daten sind im Internet über $<$ http://dnb.d-nb.de> abrufbar.

2. Auflage 2010

Alle Rechte vorbehalten

(C) Gabler Verlag | Springer Fachmedien Wiesbaden GmbH 2010

Lektorat: Stefanie A. Winter

Gabler Verlag ist eine Marke von Springer Fachmedien.

Springer Fachmedien ist Teil der Fachverlagsgruppe Springer Science+Business Media.

www.gabler.de

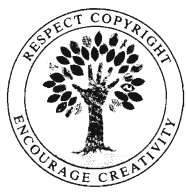

Das Werk einschließlich aller seiner Teile ist urheberrechtlich geschützt. Jede Verwertung außerhalb der engen Grenzen des Urheberrechtsgesetzes ist ohne Zustimmung des Verlags unzulässig und strafbar. Das gilt insbesondere für Vervielfältigungen, Übersetzungen, Mikroverfilmungen und die Einspeicherung und Verarbeitung in elektronischen Systemen.

Die Wiedergabe von Gebrauchsnamen, Handelsnamen, Warenbezeichnungen usw. in diesem Werk berechtigt auch ohne besondere Kennzeichnung nicht zu der Annahme, dass solche Namen im Sinne der Warenzeichen- und Markenschutz-Gesetzgebung als frei zu betrachten wären und daher von jedermann benutzt werden dürften.

Umschlaggestaltung: KünkelLopka Medienentwicklung, Heidelberg Druck und buchbinderische Verarbeitung: STRAUSS GMBH, Mörlenbach Gedruckt auf säurefreiem und chlorfrei gebleichtem Papier Printed in Germany

ISBN 978-3-8349-1873-4 


\section{Vorwort zur 2. Auflage}

Die drei thematischen Schwerpunkte dieses Buches Motivieren, Delegieren und Kritisieren treffen die entscheidenden Situationen in Unternehmen und Verwaltungen. Gerade in Zeiten einer sich verschärfenden Krise kommt dem Verhalten der Führungskräfte eine Schlüsselfunktion zu. Märkte sind in Bewegung, Gewichte verschieben sich. Dies gilt innerhalb der Organisationen, wie auch in deren Außenbeziehungen. Wer seinen Verantwortungsbereich jetzt geschickt steuert, sichert leicht Wettbewerbsvorteile und Marktanteile, die in wenigen Jahren überdurchschnittliches Wachstum ermöglichen.

Bei den derzeitigen wirtschaftlichen Rahmenbedingungen spielt neben der Motivation auch der Umgang mit Widerständen eine wesentliche Rolle. Eine sturmerprobte Mannschaft gilt es zusammenzuhalten, statt gute Kräfte an den Wettbewerber zu verlieren. Der Know-how Abfluss ist für viele Unternehmen gerade in der jetzigen Situation ein schwer zu handhabendes Thema, das nur in intensiver, vertrauensvoller und transparenter Zusammenarbeit zwischen Führungskraft und Mitarbeiter bewältigt werden kann.

Deshalb habe ich für die zweite Auflage das erfolgreiche Konzept des Buches um ein wichtiges Kapitel erweitert. Der erfolgreiche Umgang mit Widerstand in Unternehmen und Verwaltungen ist für viele Führungskräfte ein wichtiger Bestandteil der Führungsarbeit, um auch in Krisensituationen die Mitarbeiter hochgradig arbeitsfähig zu halten.

Der Umgang mit Widerstand wird auch über die wirtschaftliche Krise hinaus ein aktuelles Thema bleiben.

Die vielen positiven Rückmeldungen aus Unternehmen und Verwaltungen zu den Inhalten der ersten Auflage haben mich bestärkt, die anderen Kapitel des Buches unverändert zu lassen. Die Inhalte gehören in das Methodenrepertoire aller, die mit Führungsfragen in Berührung kommen. Dabei werden Führende wie Geführte gleichermaßen profitieren. 


\section{Vorwort zur 2. Auflage}

Ich freue mich auf den intensiven Austausch mit den Lesern und wünsche Ihnen weiterhin viele nützliche Anregungen und Impulse durch dieses Buch.

Leingarten, im April 2010

Matthias Dahms 


\section{Vorwort}

Seit mehr als fünfzehn Jahren begleitet unser Berater- und Trainerteam Führungskräfte aus Wirtschaft, Politik, Schule und Verwaltung. Während dieser Zeit beobachten wir mit Unbehagen, dass die Fachliteratur gleichsam explodiert ist. Der Leser dürstet scheinbar nach nützlichen Orientierungspunkten und droht in der Informationsflut zu ertrinken. Dicke Wälzer stehen den Leitenden zur Seite und werden oft nach ein paar Seiten frustriert zur Seite gelegt, weil die tägliche Führungspraxis nicht so gemanagt werden kann, wie die bunt bedruckten Seiten vorgeben. Entweder werden lebensfremde Theorien mit vielen Parametern entwickelt, schick in der wissenschaftlichen Diskussion, aber meist untauglich in der Praxis. Oder es wird ein trivialer Rezeptkasten vorgestellt, der der vielfältigen Führungspraxis nicht gerecht wird.

Unser Anspruch ist es, mit diesem Buch einen praktischen Beitrag zu leisten, der den Leitenden die tägliche Führungsarbeit erleichtert. Dabei stehen drei Themenfelder in Vordergrund:

Motivieren

$>$ Delegieren

- Kritisieren

Führungskräfte aller Ebenen werden zunehmend stärker gefordert und belastet. Sie brauchen solide Fähigkeiten in diesen drei Bereichen. Die Erwartungen, die an die Führungskräfte gestellt werden, sind hoch: Sich ändernde Marktbedingungen lösen erheblichen Gestaltungsdruck aus. Überall wird verändert, Pläne sind oft schon veraltet, bevor sie realisiert werden. Die Halbwertzeit des Wissens sinkt und damit auch die Halbwertzeit der Erfahrungen und Traditionen, die in der Vergangenheit Erfolge sicherstellten. Gerade heute sind deshalb Führungspersönlichkeiten gefragt, die auch auf stürmischer See Schiff und Mannschaft sicher führen. 


\section{Vorwort}

Führung ist die Beeinflussung einer Organisation oder eines Organisationsmitglieds, innerhalb einer Zielvereinbarung eigenverantwortlich tätig zu werden. Dabei hängt erfolgreiches Führen von sozialer, methodischer und fachlicher Kompetenz ab.

Der Ausgangspunkt für unsere Empfehlungen sind die Leistungsfähigkeit und Leistungsbereitschaft der Mitarbeiter. Diese zu entfalten ist ein Hauptanliegen dieses Buches. Neben der Fähigkeit, Ziele zu setzen und zu erreichen, spielt die Selbstmotivation eine wichtige Rolle. Nur wer seine Arbeit gerne macht, kann Überdurchschnittliches leisten. Außerdem erhalten Sie nützliche Strukturen für Kritikgespräche mit Mitarbeitern, Kollegen und Vorgesetzten. Dabei werde ich stets aus einem praktischen Blickwinkel beleuchten und für häufig auftretende Schwierigkeiten Lösungen anbieten.

Die Lektüre dieses Buches bietet Ihnen konkrete Anregungen für schwierige Führungssituationen. Ich wünsche Ihnen viel Erfolg bei der Lektüre und der Umsetzung in Ihre Führungspraxis. Es lohnt sich, seine Führungsfähigkeiten zu entwickeln, denn nach wie vor wird dieser Engpassfaktor exzellent honoriert.

Der Text verwendet die maskuline Wortform (Mitarbeiter, Chef, Kollege). Die Bezeichnung bezieht die weibliche Form mit ein und wurde aus Gründen der vereinfachten Lesbarkeit gewählt.

Herzlichen Dank an alle, die mich zu diesem Buch gedrängt haben, denn nur dadurch ist diese Sammlung praktischen Führungswissens entstanden. Ein Dank auch an alle, die mich bei der Textarbeit und der Recherche unterstützt haben. Insbesondere danke ich meiner Frau Katja Dahms, die die notwendigen Freiräume geschaffen hat. Außerdem danke ich Frau Barbara Krohn, Herrn Holger Nees und Herrn Alexander Volpert, die manchen Textentwurf korrigiert haben.

Leingarten, im November 2007

Matthias Dahms 


\section{Inhaltsverzeichnis}

Vorwort zur 2. Auflage

Vorwort

1. Führungsmodell__ 13

1.1 Kernbegriffe des Führungsmodells __ 14

1.2 Implementierung des Führungsverständnisses _ 20

2. Delegations- und Führungsverhalten 23

2.1 Leistungsfähigkeit und Leistungsbereitschaft___ 23

2.2 Mitarbeitertyp: Der Neue __ 26

2.3 Mitarbeitertyp: Der Veränderungsverlierer___ 31

2.4 Mitarbeitertyp: Der Vermeider __ 38

2.5 Mitarbeitertyp: Der Leistungsträger ___ 43

2.6 Mitarbeitertyp: Der Mitmacher___ 49

2.7 Die Mitarbeitertypen im Karriereverlauf __ 53

3. Kritik als Führungsinstrument

3.1 Ziel des Kritikgespräches _ 55

3.2 Angst vor dem Kritikgespräch

3.3 Der Kritikstau und seine Folgen___ 56

3.4 Voraussetzungen für ein erfolgreiches Kritikgespräch ___ 57

3.5 Das Kritikgespräch _ 64

3.6 Schwierige Situationen im Kritikgespräch ___ 71

3.7 Chancen durch ein erfolgreiches Kritikgespräch __ 84

4. Weitere Werkzeuge zum Umgang mit Regelverstößen ___ 87

$\begin{array}{ll}4.1 \text { Sichtbare Kontrollen } & 87\end{array}$ 
4.2 Feedback geben 89

4.3 Wunsch oder Bitte formulieren 92

4.4 Hinterfragen des nicht regelgerechten Verhaltens 93

4.5 Vorbildfunktion 94

4.6 Anordnung geben 97

4.7 Beteiligung 98

5. Wege zur Selbstmotivation 101

5.1 Selbstbewusstsein stärken 103

5.2 Mit Zielen zum dauerhaften Erfolg 108

5.3 Fördern Sie Ihr persönliches Durchhaltevermögen 121

5.4 Mobilisieren Sie Ihre Leistungsreserven 125

5.5 Aktiv sein: „Sich regen bringt Segen“ 130

5.6 Handeln hat oberste Priorität 137

6. Wege zur Motivation anderer Menschen

6.1 Erinnern Sie den Mitarbeiter an seine in der Vergangenheit erzielten Erfolge

6.2 Machen Sie ihm seine Stärken bewusst 140

6.3 Vereinbaren Sie gemeinsam Ziele 140

6.4 Belohnen Sie den Mitarbeiter 147

6.5 Arbeiten Sie mit der wertschätzenden Fallschirmtechnik 148

6.6 Positive Fremdsuggestionen 153

6.7 Fördern Sie die Kreativität 155

6.8 Das persönliche Erfolgstagebuch 159

6.9 Integrieren Sie den Mitarbeiter in Ihr eigenes Motivationsnetzwerk

7. Widerständen konstruktiv und souverän begegnen

7.1 Woran können Sie Widerstände erkennen? 164

7.2 Welche Ursachen lassen sich für Widerstände identifizieren? 166

7.3 Wie können Sie mit Widerständen konstruktiv umgehen? 169

7.4 Wie können Sie Widerständen vorbeugen? 175 
8. Mitarbeitergespräche als Motivationsund Führungsinstrument 179

8.1 Inhalte des Mitarbeitergespräches 180

8.2 Mitarbeitergespräch und Personalbewertung 183

8.3 Mitarbeitergespräche und Zielvereinbarungen 183

Schlussbemerkung 187

Abbildungsverzeichnis 189

Literaturverzeichnis 191

Stichwortverzeichnis 193

Der Autor 196 